\title{
UNA APROXIMACIÓN DE LA EVALUACIÓN DEL AMBIENTE FÍSICO, LA CARGA FÍSICA Y MENTAL DE LOS CONDUCTORES DE BUS URBANO DE PASAJEROS
}

Recibido: septiembre del 2018

Aceptado: abril del 2019

Armando Engels Durán Urón ${ }^{1}$, Angie Isabel Meléndez Serrano²

\section{Resumen}

Este estudio se realizó con el fin de conocer si la carga mental y el ambiente físico tienen una valoración diferente a la carga física en los conductores de buses urbanos de pasajeros. Igualmente, el estudio permitió conocer realidades laborales en un contexto productivo, con el fin de velar por el cumplimiento de la normativa laboral y asegurar la debida protección de los trabajadores. Para esta investigación se utilizó el método LEST, el cual permitió evaluar las condiciones de trabajo de la forma más objetiva y global posible, al mostrar cada una de las situaciones consideradas en el puesto como satisfactoria, molesta o nociva. Para comprobar las condiciones de trabajo, se evaluaron 16 variables agrupadas en cinco dimensiones: entorno físico, carga física, carga mental, aspectos psicosociales y tiempo de trabajo. Se concluye que las variables que requieren de mayor atención son el ambiente físico, la carga mental y el tiempo de trabajo.

Palabras clave: ambiente físico, carga física, carga mental, conductores de buses.

1 Ingeniero industrial, magíster en Prevención de Riesgos Laborales, doctor en Ciencias Gerenciales. Profesor de la Universidad del Atlántico. Correo: armandoduran@mail. uniatlantico.edu.co

2 Ingeniera agroindustrial, magíster en Seguridad Alimentaria y Nutricional. Coordinadora de programas técnicos de la Universidad del Atlántico. Correo: amelendezserrano@mail. uniatlantico.edu.co 


\section{AN APPROXIMATION TO THE EVALUATION OF THE PHYSICAL ENVIRONMENT, AND THE PHYSICAL AND MENTAL BURDEN OF BUS DRIVERS}

Armando Engels Durán Urón ${ }^{1}$, Angie Isabel Meléndez Serrano²

\section{Abstract}

The purpose of this study was to know if the mental burden and the physical environment have a different value from the physical burden in bus drivers in the city. Likewise, the study allowed us to know about their work realities in a productive context, in order to ensure compliance with work laws and with the protection of workers. We used the LEST method, which allowed us to evaluate work conditions as objectively and globally as possible, by showing each one of the situations at the workplace as either satisfactory, disturbing or harmful. Thus, 16 variables were evaluated and they were groupd in 5 dimensions: physical setting, physical burden, mental burden, psychosocial aspects and time of work. We concluced that the variables that require more attention are physical setting, mental burden and time of work. 


\section{UMA APROXIMAÇÃO DA AVALIAÇ̃̃OO DO AMBIENTE FÍSICO, DA CARGA FÍSICA E MENTAL DOS MOTORISTAS DE ÔNIBUS URBANO DE PASSAGEIROS}

Armando Engels Durán Urón ${ }^{1}$, Angie Isabel Meléndez Serrano ${ }^{2}$

\section{Resumo}

Este estudo foi realizado com o objetivo de verificar se a carga mental e o ambiente físico têm um valor diferente ao da carga física nos motoristas de ônibus urbanos de passageiros. Além disso, permitiu conhecer realidades no âmbito profissional em um contexto produtivo, a fim de zelar pelo cumprimento da lei trabalhista e garantir a devida proteção dos trabalhadores. Para esta pesquisa, foi utilizado o método LEST, que permitiu avaliar as condições de trabalho da forma mais objetiva e global possível, ao mostrar cada uma das situações consideradas no posto como satisfatória, incômoda ou nociva. Para comprovar as condições de trabalho, foram avaliadas 16 variáveis, agrupadas em cinco dimensões: ambiente físico, carga física, carga mental, aspectos psicossociais e tempo de trabalho. Conclui-se que as variáveis que exigem mais atenção são o ambiente físico, a carga mental e o tempo de trabalho. 


\section{Introducción}

El sector de transporte urbano de pasajeros, es una actividad poco explorada en su dimensión laboral, con complejas formas de organización del trabajo ajustable a las necesidades del mercado, y que involucra un importante factor humano, representado en los conductores de buses.

La literatura relacionada con bus urbano de pasajeros muestra que los riesgos de tipo físico intervienen de manera considerable en la labor de conducción de vehículo de pasajeros, específicamente que el disconfort o incomodidad térmica se asocia con una mayor prevalencia de hipertensión (1). Igualmente, es una problemática manifiesta, la exposición a vibraciones de cuerpo entero y la enfermedad lumbar(2), (3). y es posible encontrar que los conductores de autobuses están potencialmente expuestos a niveles de vibración diarios más altos que los recomendados, especialmente en ciertos tipos de carretera (4)

Por otra parte, un estudio de la "calidad del trabajo" y "buenos trabajos", encontró que la calidad del trabajo de los conductores de buses era significativamente más baja que la de un grupo de no conductores en la misma organización (5). Otro aspecto importante es la fatiga, en los conductores de autobuses se asocia a 228 condiciones de trabajo estresantes y agotadoras (6).Y,su exposición a condicio- nes de trabajo inadecuadas que parecen interferir en la salud ocupacional. (7)

En cuanto a factores contribuyente a la posibilidad de accidentarse están las condiciones de trabajo relacionadas con el estrés las cuales son factores predictivos relevantes de conducción arriesgada, igualmente la fatiga es el mecanismo que vincula otro tipo de estrés relacionado con las condiciones de trabajo (tensión laboral y bajo apoyo social) con conducción arriesgada.(8)

Otro componente que contribuye a las condiciones laborales son los usuarios, debido a que su comportamiento cívico, influencia el grado de satisfacción laboral de los conductores (9). De la misma forma, los riesgos de tipo psicosocial asociados a la labor de conducción son el estrés, la ansiedad, la depresión y la insatisfacción con las condiciones laborales (10). Igualmente, los conductores de buses están expuestos a una serie de condiciones desfavorables que pueden poner en peligro su salud y generar algún tipo de estrés (11). Específicamente, en Colombia la prevalencia de estrés relacionado con el trabajo y el agotamiento, están significativamente elevados en los conductores de autobuses (12).

No menos importante es la postura de trabajo, presentan un menor riesgo postural los conductores de buses de tipo automático que aquellos que conducen los de tipo mecánico (13). En relación 
con las dimensiones físicas de los conductores y el diseño dimensional de las cabinas de autobús, existe el riesgo de daño musculoesquelético en los hombros y, principalmente, en el cuello, debido a los movimientos de extensión frecuente al mirar por el retrovisor central (13) igualmente, molestias en la espalda y extremidades superiores (14)(15).

Lo anterior, motiva la realización de este trabajo, en una ciudad del caribe colombiano buscando valorar la carga física y mental, al analizar el puesto de trabajo de los conductores de buses de una empresa de transporte urbano de pasajeros mediante el método LEST, que revisa 16 variables agrupadas en 5 dimensiones asociadas a la carga física y mental de los trabajadores durante el recorrido de la ruta y de 3 tipos de vehículos; bus, buseta y microbús. El estudio agrupa los factores de riesgo más relevantes, indicando cuál de las variables tiene mayor calificación, analiza sus causas y al final, mostrará necesidades de nuevos estudios detallados para esta actividad económica en Colombia. Por último, se muestran posibles causas y recomendaciones que permitirían mejorar las condiciones laborales de los conductores de buses.

\section{Materiales y métodos}

Para la evaluación de los puestos de trabajo, se utilizó la observación directa al trabajador, acompañamiento durante la ruta del recorrido, se realizaron tres evaluaciones a tres conductores de buses de diferente capacidad, el primer vehículo apto para trasladar sentado 40 pasajeros, el segundo 50 y el tercero 18 . Los trayectos de la ruta se hicieron a lo largo y ancho de una ciudad de la costa caribe colombiana con tiempos de desplazamiento entre 2.5 y 3.5 horas, con turnos de trabajo mayor a 12 horas al día con descanso de 30 minutos entre un recorrido y otro.

Posterior a la observación, se entrevistó al conductor, al coordinador de seguridad y salud en el trabajo de la empresa $y$ al coordinador de recursos humanos para conocer su percepción sobre las variables de análisis que propone el método LEST. Los instrumentos utilizados para la realización del estudio fueron cámaras de video, el SV84 medidor de vibración humana, monitor de estrés térmico QUESTEMP 34 y dosímetro marca Quest Tecnologies modelo: EDGE.

Se recogió la información usando la hoja de observación propuesta en el número 175 de la Nota técnica de prevención (16). Para la evaluación del puesto de trabajo se observó la ejecución de la labor en todo su recorrido, y posteriormente, se realizó entrevista para corroborar la carga de trabajo, se utilizaron instrumentos de filmación de las tareas. Las evaluaciones se realizaron en el horario pico de 10:00 am a 2:00 pm hora de mayor temperatura, ruido y movimiento de pasajeros.

Se seleccionó el método LEST porque permite hacer una evaluación global de la carga física y mental del puesto de 
trabajo, que cuente con la participación de miembros de recursos humanos, de seguridad y salud en el trabajo y de trabajadores de bajo nivel de escolaridad. Para el procesamiento de los datos se tabularon tomando como guía los números 175, 626 y 627 de las notas técnicas de prevención.(17) y (18) . Para el análisis de los resultados, se hizo un resumen de los resultados obtenidos tabulando el factor de disconfort o cada variable con su calificación por puesto de trabajo. Posteriormente, se tabularon los datos, graficaron y analizaron a la luz del método LEST y se muestrearon las condiciones de trabajo de los conductores, Por último, se analizaron las principales causas de cada factor de disconfort y se propusieron medidas preventivas, que mejoren la calidad de vida del conductor.

Para describir brevemente el método Lest (19) desarrollado por F. Guélaud, M.N. Beauchesne, J. Gautrat y G. Roustang, miembros del Laboratoire d'Economie et Sociologie du Travail (L.E.S.T.), y que busca evaluar las condiciones de trabajo de la forma más objetiva y global posible, estableciendo un diagnóstico final que indique si cada una de las situaciones consideradas en el puesto es satisfactoria, molesta o nociva. El método es de carácter global considerando cada aspecto del puesto de trabajo de manera general. No se profundiza en cada uno de esos aspectos, si no que se obtiene un tamizaje que permite establecer si se requiere un análisis más profundo con métodos específicos.

El objetivo es del método, es evaluar el conjunto de factores relativos al contenido del trabajo que pueden tener repercusión tanto sobre la salud como sobre la vida personal de los trabajadores. La información que es preciso recoger para aplicar el método tiene un doble carácter objetivo-subjetivo. Por un lado, se emplean variables cuantitativas como la temperatura o el nivel sonoro, y por otra, es necesario recoger la opinión del trabajador respecto a la labor que realiza en el puesto para valorar la carga mental o los aspectos psicosociales del mismo. Es pues necesaria la participación en la evaluación del personal implicado.

Por ultimo para aplicar el método LEST debe recogerse la información suficiente requerida para valorar 5 dimensiones que considera. Cada dimensión se subdivide en una serie de variables mostrada en la Tabla 1."

Tabla 1. Dimensiones y variables en LEST 


\begin{tabular}{|c|c|c|c|c|}
\hline Entorno físico & Carga física & Carga mental & $\begin{array}{c}\text { Aspectos } \\
\text { psicosociales }\end{array}$ & $\begin{array}{c}\text { Tiempos de } \\
\text { trabajo }\end{array}$ \\
\hline $\begin{array}{l}\text { Ambiente } \\
\text { térmico }\end{array}$ & Carga estática & $\begin{array}{l}\text { Apremio de } \\
\text { tiempo }\end{array}$ & Iniciativa & $\begin{array}{l}\text { Tiempo de } \\
\text { trabajo }\end{array}$ \\
\hline Ruido & Carga dinámica & Complejidad & Estatus social & \\
\hline Iluminación & & Atención & Comunicaciones & \\
\hline Vibraciones & & & $\begin{array}{l}\text { Relación con el } \\
\text { mando }\end{array}$ & \\
\hline
\end{tabular}

*DIEGO-MAS, JOSE ANTONIO. Análisis ergonómico global mediante el método LEST. Ergonautas, Universidad Politécnica de Valencia, 2015. Disponible online: https:/www.ergonautas.upv.es/ metodos/lest/lest-ayuda.php

En consonancia con lo anterior cuando se han recogido los datos deben consultarse una serie de tablas de puntuaciones que permiten obtener las valoraciones de cada variable y dimensión. El número de tablas que es necesario consultar es muy elevado, por lo que

Tabla 2. Puntuación de las variables en el método LEST la aplicación del método puede ser laboriosa. La valoración obtenida para cada dimensión oscila entre 0 y 10 y la interpretación de dichas puntuaciones se realiza según la Tabla 2."

\section{\begin{tabular}{l|l}
\hline Puntuación & Valoración \\
\hline
\end{tabular}}

$0,1,2$

$3,4,5$

6,7

8,9

10

\section{Situación satisfactoria}

Débiles molestias. Algunas mejoras podrían aportar más comodidad al trabajador

Molestias medias. Existe riesgo de fatiga

Molestias fuertes. Fatiga

Situación nociva

** DIEGO-MAS, JOSE ANTONIO. Análisis ergonómico global mediante el método LEST. Ergonautas, Universidad Politécnica de Valencia, 2015. Disponible online: https://www.ergonautas. upv.es/metodos/lest/lest-ayuda.php 
Por último, la valoración final se represente en forma de histograma. Esta representación gráfica permite tener una visión rápida de las condiciones de trabajo. Conociendo cuáles son los elementos más desfavorables y se pueden establecer prioridades a la hora de intervenir sobre los distintos factores evaluados".

\section{Resultados}

Se aprecia en la gráfica 1. un comportamiento muy parecido en los resultados de la evaluación del puesto de trabajo de los tres tipos de buses, las variables con calificaciones entre $6 \mathrm{y}$ 10 o aquellas causantes de molestias medias y altas para el trabajador por dimensiones son las siguientes:

En la dimensión entorno físico: las vibraciones, el ruido y el ambiente térmico. En la dimensión carga mental la atención mantenida. En la dimensión aspectos psicosociales con mayor participación la falta de iniciativa, el bajo estatus social, la poca comunicación y la escasa relación con los directivos, por último, en la dimensión el tiempo de trabajo, la Organización y la cantidad de tiempo en trabajo.

Grafico 1. Resultados evaluación de variables
En la dimensión carga física se muestran calificaciones por debajo de 6 . No se considera la carga dinámica, debido a que el trabajador conserva su postura durante casi todo su recorrido, Las causas asociadas a las variables de mayor puntuación se resumen en la siguiente tabla2.

En general, se aprecia que los conductores evaluados con diferentes tipos de buses con rutas y tiempos de desplazamiento diferente presentan la calificación más alta de las variables propuestas por el método de estudio, están incluidas en las dimensiones entorno físico, carga mental, aspectos psicosociales y tiempos de trabajo. A diferencia de la dimensión carga física que no tiene calificación considerable. 
Gráfico de tres puestos de trabajo por tipo de bus-dimensión-variable

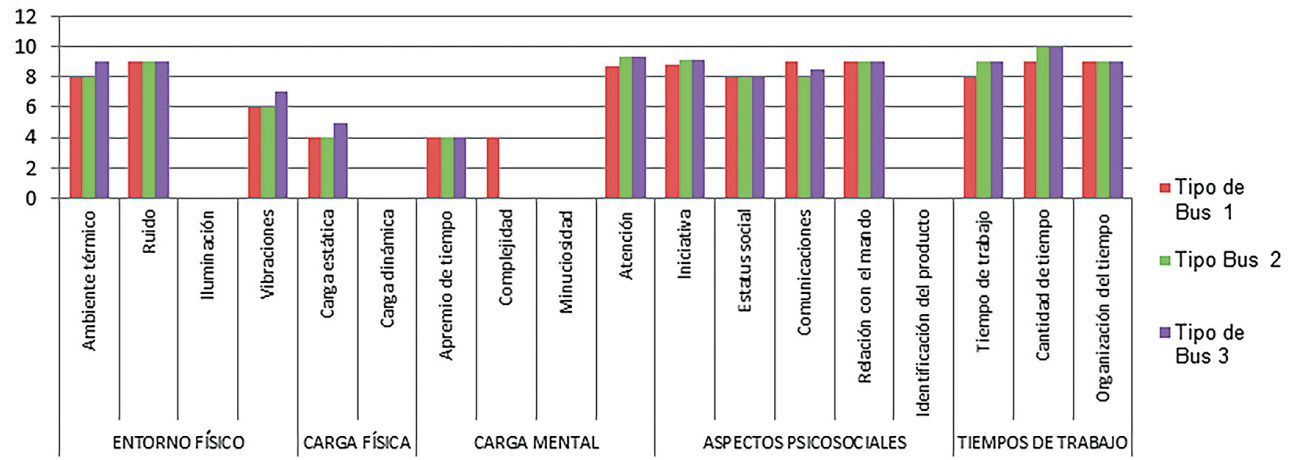

\section{Discusión}

Este estudio, muestra que la carga mental y el ambiente físico están mayormente valoradas que la carga física para los conductores de bus urbano de pasajeros para una ciudad de ambiente caluroso como las ubicadas en la costa norte colombiana, coherente con lo indicado por Aquino Jael y et al, en su estudio que muestran un diagnóstico preocupante sobre la existencia de riesgos físicos y psicosociales a los que están expuestos

\section{Agradecimientos}

Se agradece a todas las personas que estuvieron abiertas, de manera sincera y participativa en la realización de este estudio.

- Los autores manifiestan su consentimiento para publicar el artículo, los conductores de buses. Igualmente, muestra la necesidad en investigar detalladamente los factores de riesgos psicosociales asociados a las largas jornadas de trabajo y la atención mantenida potencializados con estresores de tipo físico como el ruido la temperatura y las vibraciones de cuerpo entero.

- También que no existe conflicto de intereses entre los autores $\mathrm{u}$ otra persona.

- Por último, que el documento no se ha publicado previamente, ni se encuentra en revisión. 


\section{Referencias bibliográficas}

(1). Pimenta, A. and Assunção, A. (2015). Thermal discomfort and hypertension in bus drivers and chargers in the metropolitan region of Belo Horizonte, Brazil. Applied Ergonomics, 47, pp.236-241.

(2) Jonsson, P., Rynell, P., Hagberg, M. and Johnson, P. (2014). Comparison of whole-body vibration exposures in buses: effects and interactions of bus and seat design. Ergonomics, 58(7), pp.1133-1142.

(3) Tore'n, Kjell, Maria Albin, and Bengt Ja"rvholm. 2012. "Systematiska kunskapso"versikter. 2, Exponering fo" $r$ helkroppsvibrationer och uppkomst av la"ndryggssjuklighet." Arbete och ha"Isa. 2012;46(2), Go"teborgs Universitet.

(4). C. A. Lewis, P. W. Johnson, Whole-body vibration exposure in metropolitan bus drivers, Occupational Medicine, Volume 62, Issue 7, October 2012, Pages 519-524.

(5) Jones W, Haslam R, Haslam C. Measuring job quality: A study with bus drivers. Applied Ergonomics. 2014;45(6):1641-1648.

(6) Filho A, Rocha M, Oliveira Á, Santos U, Costa J, Jesus J. Working Conditions of Bus Drivers in a Large Brazilian Metropolis. Procedia Manufacturing. 2015;3:2505-2509.

(7) Aquino Jael Maria de, Gomes de Medeiros Sílvia Elizabeth, Mata Ribeiro Gomes Betânia da, Batista Ferreira e Pereira Emanuela, Brandão Neto Waldemar, Gomes Terra Marlene. Condiciones de trabajo en conductores de autobús: de servicio público a fuente de riesgo. Index Enferm [Internet]. 2017 Jun [citado 2019 Mayo 07] ; 26(1-2 ): 34-38.

(8)Sergio A.UsecheaViviola GómezOrtizbBoris E.Cendalesc. Stress-related port (BRT) drivers. Accident Analysis \& Prevention 2019; Volume 104, July 2017

(9) Hinestroza M, Campoverde M. incidencias de los comportamientos cívicos de los usuarios sobre la satisfacción laboral en los conductores del sistema metrovía de la ciudad de guayaquil, 2017-08-13

(10). Bonilla RLR, Gafaro rai. condiciones laborales y riesgos psicosociales en conductores de transporte público. Revista Cubana de Salud y Trabajo 2017; 18 (2); 2017.

(11). Catalina González Penagos, Diana Verónica Giraldo López, Melissa Cano Gómez. factores laborales y estrés percibido en los conductores de buses medellín, 2017 .

(12). Useche, Sergio \& Cendales, Boris \& Alonso, Francisco \& Serge, Andrea Cecilia. (2017). Comparing Job Stress, Burnout, Health and Traffic Crashes of Urban Bus and BRT Drivers. American Journal of Applied Psychology. 5. 25-32. 10.12691/ajap-5-1-5.

(13). Martha Yaneth,Herazo Beltrán, Raul Polo-Gallardo, Roberto Rebolledo. Inferencias en el riesgo postural y en la percepción de molestias músculoesqueléticas en conductores de autobuses de transporte urbano con transmisión mecánica o automática. Archivos Venezolanos de Farmacologia y Terapeutica $2017 ;:$.

(14). Verónica Rayo, Érika Arias. Especificaciones Ergonómicas para el Diseño Dimensional de la Cabina de Conductor de Autobús Urbano. Archivos Venezolanos de Farmacologia y Terapeutica 2017.

(15) Yasobant, Sandul \& Chandran, Madhan \& Manikanta Reddy, Endreddy. (2015). Are Bus Drivers at an Increased Risk for Developing Musculoskeletal Disorders? An Ergonomic Risk Assessment Study. Journal of Ergonomics. S3. 1-5. 10.4172/2165-7556.S3-011. 
(16) NTP 175 de 1986, Evaluación de las Condiciones de Trabajo: el método L.E.S.T.

(17) NTP 626 de 2003, Método LEST (I): aplicación a una empresa de empaquetado.

(18) NTP 627 de 2003, Método LEST (I): aplicación a una empresa de empaquetado. Recuperado en [30 de agosto 2017] de 6.

(19) Diego-Mas, Jose Antonio. Análisis ergonómico global mediante el método LEST. Ergonautas, Universidad Politécnica de Valencia, 2015. Disponible 